\title{
SOME STRATEGIES FOR HARVESTING A SINGLE SPECIES
}

\author{
G. W. SWAN* \\ Theoretical Biology Group, \\ University of Utrecht, \\ Netherlands
}

The $n$-stage harvesting strategy of Elizarov and Svirezhev is examined. As a result, some important new features appear. A discussion is presented on whether or not one should harvest a species at one time stage or wait until a later time. The paper is concerned with contributions which are primarily mathematical formulations and results for continuous, as well as discrete time, logistic growth of a single species being harvested. Age class structure is ignored.

Introduction. The problem of harvesting members of a biological species is of considerable interest in many areas of application. For example, one may be interested in culling from a population of microorganisms in a chemostat (D'Ans, Kokotović and Gottlieb, 1971), controlling a lethal growth process (Cherniavsky and Taylor, 1972), or it may be desirable to destroy members of a bacterial population by the administration of a drug (Bellman and Kalaba, 1960), or one may seek to remove insect pests from damaging a crop (Becker, 1970; Shoemaker, 1973). A brief survey of some of the areas of biological control is given by Jaquette (1972). However, there are many more applications and the literature is quite extensive.

A particularly interesting paper by Elizarov and Svirezhev (1972, published in Russian in 1970) appears to be quite unknown to workers in this general area of harvesting and control. The first part of their paper is concerned with the optimal culling in a population described by Volterra's model. Their problem,

* Present address: Department of Mechanical and Aerospace Engineering, University of Arizona, Tueson, AZ 85721. 
fundamentally, is very interesting not only from the mathematical but also from the biological point of view. The objective of the present paper is (i) to present some of their work, (ii) to clarify the mathematical and biological nature of the harvesting processes and the implementation of alternate, viable, strategies and (iii) to extend their work to deal with the harvesting of populations that appear to be modeled by discrete time logistic growth models.

Continuous Time Logistic Growth. Assume that a species grows according to the Verhulst-Pearl logistic model (Verhulst, 1845, 1847; Pearl, 1924):

$$
\dot{N}=(a-b N) N, \quad a, b \text { constants, }
$$

where $N \equiv N(t)$ denotes the number of individuals in the population at time $t$, $a(>0)$ is the specific growth factor, and $b$ is a measure of the overcrowding of the species. (The disadvantage of this model is that it does not provide information concerning the age distribution of the population.) The logistic model has enjoyed a popularity with many biologists because it can be used to fit many growth curves $(\dot{N}>0)$ and also many mortality curves $(\dot{N}<0)$. With $N=$ $N_{0}$, the initial prescribed size of the population at time $t_{0}$, and under the assumption that $N_{0}$ is sufficiently large enough in order to talk sensibly about the derivative $\dot{N}$, then (1) has the solution

$$
N(t)=a N_{0} \mathrm{e}^{a\left(t-t_{0}\right)} /\left\{a+b N_{0}\left[\mathrm{e}^{\alpha\left(t-t_{0}\right)}-1\right]\right\} .
$$

For growth, the species increases monotonically from $N_{0}$ to the carrying capacity $N_{c}(=a / b)$ as $t \rightarrow \infty, N_{0} \leq N \leq N_{c}$, whereas if $N_{0}>N_{c}$ the curve of $N$ vs time ( $t$ as abscissa) decreases monotonically to $N_{c}$ as $t \rightarrow \infty$. The quantity $N_{c}$ is a stable equilibrium point of the differential equation (1).

For each of the harvesting strategies considered in this paper it is assumed that when a population is harvested it is done so instantaneously.

The Strategy of Elizarov and Svirezhev. Consider growth of a species according to (1), (2). Our first decision is to work with a definite time horizon of fixed length $T=t_{n}-t_{0}$, where $t_{n}$ denotes the final (known or specified) time, and the subscript $n$ is regarded as fixing the end of the time interval.

A one-stage harvesting process is defined as follows: Let the population commence with $N_{0}$ and grow to $N\left(t_{n}\right)$ at $t_{n}$ at which time all individuals are harvested. Consequently the maximum yield in this process is

$$
y_{1}=N\left(t_{n}\right) \text {. }
$$

A two-stage harvesting process is defined as follows. Retain the same fixed time horizon as in the one-stage process. Divide $T$ into two equal time intervals such that $t_{n}-t_{1}=t_{1}-t_{0}$, and define

$$
\lambda=\lambda\left(t_{1}-t_{0}\right)=\exp \left[a\left(t_{1}-t_{0}\right)\right] .
$$


Let the population commence with $N_{0}$ at $t_{0}$ and grow to the number $N_{1}$ at time $t_{1} ; N_{1} \equiv N\left(t_{1}\right)$. Harvest a fraction $k(0<k<1)$ of $N_{1}$ at this time so that the new initial population is $(1-k) N_{1}$ at the beginning of the second time interval. The species now grows until $t_{n}$ at which time all individuals are harvested. For this two-stage process (stage 1 is the time $t_{1}$, stage 2 is the time $t_{n}$ ) the yield is

$$
\begin{aligned}
y(k) & =k N_{1}+N\left(t_{n}\right) \\
& =k N_{1}+a(1-k) N_{1} \lambda /\left[a+b(1-k)(\lambda-1) N_{1}\right] .
\end{aligned}
$$

Note that $k$ may now be a continuous variable or it may be a discrete variable. In each case our objective is to obtain the maximum yield

$$
y_{2}=\max _{0<k<1} y(k) .
$$

It is convenient to refer to $k$ as an influence variable.

For $k$ continuous it is meaningful to use differential calculus and, regarding (5) as a function of the single variable $k$, it is straightforward to verify that

maximizes $y(k)$ and

$$
k=1-a / b\left(\lambda^{1 / 2}+1\right) N_{1}
$$

$$
\begin{aligned}
y_{2} & =N_{1}+a\left(\lambda^{1 / 2}-1\right) / b\left(\lambda^{1 / 2}+1\right) \\
& =a N_{0} \lambda /\left[a+b N_{0}(\lambda-1)\right]+a\left(\lambda^{1 / 2}-1\right) / b\left(\lambda^{1 / 2}+1\right) .
\end{aligned}
$$

The biological interpretation of (6) is as follows. Since $a, b, \lambda, N_{0}$ are fixed, so also is $N_{1}$. If $a / b\left(\lambda^{1 / 2}+1\right) N_{1} \geqslant 1$ then one should not harvest whereas one can harvest whenever $a / b\left(\lambda^{1 / 2}+1\right) N_{1}<\mathrm{I}$. Also, $N\left(t_{n}\right)=a \lambda^{1 / 2} / b\left(\lambda^{1 / 2}+1\right)$.

Now consider a three-stage harvesting. Divide the fixed time horizon into three equal parts by $t_{1}, t_{2}$ such that $t_{1}-t_{0}=t_{2}-t_{1}=t_{n}-t_{2}$ and define $\lambda$ by (4). For the present situation the actual numerical value of $\lambda$ is smaller than in the two-stage process. The initial population is $N_{0}$ and the species grows to the number $N_{1}$ at $t_{1}$. A fraction $k_{1}\left(0<k_{1}<1\right)$ of $N_{1}$ is taken and the new starting population is $\left(1-k_{1}\right) N_{1}$ at the beginning of the second time interval. The species grows to the number $N_{2}$ at time $t_{2}$ and a fraction $k_{2}\left(0<k_{2}<1\right)$ of $N_{2}$ is taken. Finally, at the time $t_{n}$ all remaining individuals are harvested. It follows, for the three-stage harvesting process, that the yield

where

$$
y\left(k_{1}, k_{2}\right)=k_{1} N_{1}+\varphi\left(k_{1}, k_{2}\right)+\psi\left(k_{1}, k_{2}\right),
$$

$$
\begin{aligned}
& \varphi\left(k_{1}, k_{2}\right)=a \lambda N_{1} k_{2}\left(1-k_{1}\right) /\left[a+b(\lambda-1)\left(1-k_{1}\right) N_{1}\right] \\
& \psi\left(k_{1}, k_{2}\right)=\frac{a \lambda^{2}\left(1-k_{1}\right)\left(1-k_{2}\right) N_{1}}{a+b(\lambda-1)\left(1-k_{1}\right) N_{1}+b N_{1}\left(\lambda^{2}-\lambda\right)\left(1-k_{1}\right)\left(1-k_{2}\right)} .
\end{aligned}
$$


Our objective is to obtain the maximum yield

$$
y_{3}=\max _{\substack{0<k_{1}<1 \\ 0<k_{2}<1}} y\left(k_{1}, k_{2}\right) .
$$

Assume that the influence variables are continuous. It follows from this that necessary conditions for a local extremum of $y\left(k_{1}, k_{2}\right)$ are given by the simultaneous solution of $\partial y / \partial k_{1}=\partial y / \partial k_{2}=0$. The second of these gives

$$
1-k_{2}=\left[a+b\left(1-k_{1}\right)(\lambda-1) N_{1}\right] / b N_{1} \lambda\left(1-k_{1}\right)\left(\lambda^{1 / 2}+1\right) .
$$

Substitution of this result into $\partial y / \partial k_{1}=0$ gives

and hence

$$
k_{1}=1-a / b\left(\lambda^{1 / 2}+1\right) N_{1},
$$

$$
k_{2}=1-\lambda^{-1 / 2} \text {. }
$$

The mathematical form for $k_{1}$ is the same as that for $k$ given by (6) for the twostage process but the numerical values are different since $\lambda$ does not have the same numerical value.

It is a more algebraically complex problem to verify that (9), (10) give a local maximum for the yield $y\left(k_{1}, k_{2}\right)$. Here

$$
y_{3}=N_{1}+2 a\left(\lambda^{1 / 2}-1\right) / b\left(\lambda^{1 / 2}+1\right),
$$

which (compare (7)) is greater than $y_{2}$. Also,

$$
N_{2}=N\left(t_{n}\right)=a \lambda^{1 / 2} / b\left(\lambda^{1 / 2}+1\right) .
$$

Now for this three-stage harvesting process, the difference

$$
N_{2}-N_{1}=a \lambda^{1 / 2}\left[a-b N_{0}\left(\lambda^{1 / 2}+1\right)\right] / b\left(\lambda^{1 / 2}+1\right)\left[a+b N_{0}(\lambda-1)\right],
$$

and at the beginning of the second time interval the initial population is $a / b\left(\lambda^{1 / 2}+1\right)$. Since $a, b, N_{0}$ and $\lambda$ are fixed, the difference

$$
a-b N_{0}\left(\lambda^{1 / 2}+1\right)\left\{\begin{array}{r}
>0 \\
\text { or }=0 \\
\text { or }<0
\end{array}\right.
$$

The top inequality corresponds to $N_{2}>N_{1}$, the equality gives $N_{2}=N_{1}$ and the bottom inequality implies $N_{2}<N_{1}$. Accordingly the assumed population model and the fixed time horizon lock into one of the three curves shown in Figure 1.

The bottom inequality in (12) is interesting. It says that, at the time $t_{1}$, the population level down to which one has harvested, namely $a / b\left(\lambda^{1 / 2}+1\right)$, is less than $N_{0}$ which is available at time $t_{0}$. This would seem to suggest that if too many individuals are harvested then not enough remain to promote the increase 
in the population and the species would therefore be driven to extinction. However, this cannot be true here for the reason that at low numbers $\left(\ll N_{0}\right)$ the assumed logistic model cannot be satisfactorily implemented because no

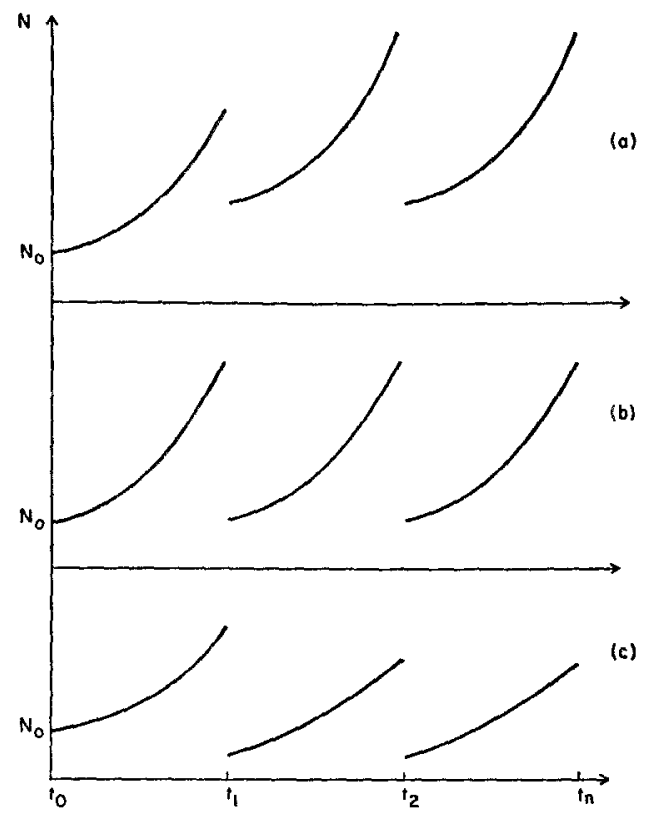

Figure 1. Three-stage harvesting process. A population follows the curves indicated by (a) when $a-b N_{0}\left(\lambda^{3 / 2}+1\right)>0$, and follows the curves (b) when $a-b N_{0}\left(\lambda^{1 / 2}+1\right)=0$, and when $a-b N_{0}\left(\lambda^{1 / 2}+1\right)<0$ it follows the curves designated by (c)

sense can be made of $\dot{N}$. It is appropriate, therefore, to introduce the critical level of population $N_{\text {crit }}$ with

$$
N_{\text {crit }}<a / b\left(\lambda^{1 / 2}+1\right)<N_{0} .
$$

If $a / b\left(\lambda^{1 / 2}+1\right)$ turns out to be less than some specified value of $N_{\text {crit }}$ then one can no longer use our assumed continuous time logistic growth model.

The n-stage harvesting process. From the earlier discussions it is apparent that, in the $n$-stage harvesting process, the following are true, when the influence variables are assumed to be continuous:

$$
\begin{gathered}
k_{1}=1-a / b\left(\lambda^{1 / 2}+1\right) N_{1}, \quad k_{2}=k_{3}=\cdots=k_{n-1}=1-\lambda^{-1 / 2}, \\
N_{2}=N_{3}=\cdots=N\left(t_{n}\right)=a \lambda^{1 / 2} / b\left(\lambda^{1 / 2}+1\right),
\end{gathered}
$$


and the maximum yield

$$
\begin{aligned}
y_{n} & =k_{1} N_{1}+k_{2} N_{2}+\cdots+k_{n-1} N\left(t_{n-1}\right)+N\left(t_{n}\right) \\
& =k_{1} N_{1}+(n-2) k_{2} N_{2}+N\left(t_{n}\right) \\
& =N_{1}+(n-1) a\left(\lambda^{1 / 2}-1\right) / b\left(\lambda^{1 / 2}+1\right) .
\end{aligned}
$$

This last result is stated by Elizarov and Svirezhev.

A check on (13) is provided by letting $n$ become infinite, for then there is the situation of continuous harvesting. For $n=T / h$, where $h$ is the length of a time interval,

$$
\begin{aligned}
y_{n}=y(h) & =\frac{a N_{0} \mathrm{e}^{a h}}{a+b N_{0}\left(\mathrm{e}^{a h}-1\right)}+\frac{T-h}{h} \frac{a}{b} \frac{\exp (0.5 a h)-1}{\exp (0.5 a h)+1} \\
& \rightarrow N_{0}+a^{2} T / 4 b, \text { as } h \rightarrow 0,
\end{aligned}
$$

which is interpreted as the maximum yield under continuous harvesting. The last displayed result is well known and can be obtained directly from the logistic equation (l).

Our objective in examining the Elizarov-Svirezhev strategy has revealed new features. Further insights are developed in the next section.

Discussion on $Y$ ield. It is important to realize that the result (13) does not imply that $y_{n}>y_{n-1}$ since the $\lambda$ used in $y_{n}$ has a smaller numerical value than the $\lambda$ used in $y_{n-1}$. The purpose of this section is to examine the maximum yields and gain insight into the harvesting strategies.

For example, consider the time interval $\left[t_{0}, t_{n}\right]$ and let the species grow from size $N_{0}$ to $N(t)$, then harvest everything at the final (fixed) time $t_{n}$. This gives the maximum take for the one stage process as

$$
Y_{1}=\frac{a N_{0} \exp \left[a\left(t_{n}-t_{0}\right)\right]}{a+b N_{0}\left\{\exp \left[a\left(t_{n}-t_{0}\right)\right]-1\right\}}=\frac{a N_{0} \lambda^{2}}{a+b N_{0}\left(\lambda^{2}-1\right)},
$$

where $\lambda$ is defined as in (4) and, here, $t_{n}-t_{0}=2\left(t_{1}-t_{0}\right)$. Now consider the two stage process with the maximum yield $y_{2}$ being given by (8). At this point $Y_{1}$ and $y_{2}$ are given in terms of the same numerical value of $\lambda$. Is $y_{2}$ greater than $Y_{1}$ ? To answer this question the difference in yields, namely $\sigma$, is constructed, and this is considered to be a function of $\lambda$. After a little algebra,

$$
\sigma(\lambda)=y_{2}-Y_{1}=N_{c}\left(K-\lambda^{3 / 2}\right)^{2}\left(\lambda^{1 / 2}-1\right) /\left(K+\lambda^{2}\right)(K+\lambda)\left(\lambda^{1 / 2}+1\right),
$$

where $K=N_{c} / N_{0}-1$. The growth of $\sigma(\lambda)$ is shown in Figure 2 for the situation when $K>1$, and for the tenable values of $\lambda(\lambda>1$ ). (When $K=1$ the two points on the $\lambda$-axis coincide and the hump vanishes. Values of $K$ 


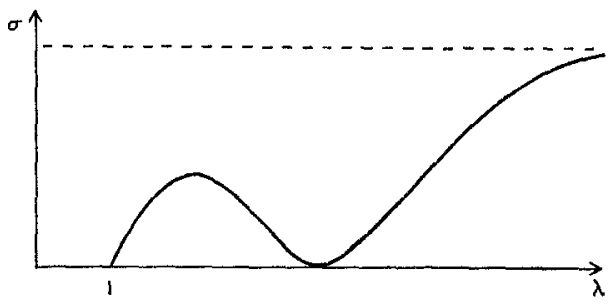

Figure 2. The difference in yields from two alternative strategies is plotted against the nondimensional parameter $\lambda$. The ordinate is given by (14)

which are less than unity are of no consequence to the analysis of (14) because $\lambda>1$.) By inspection of this figure it is apparent that one always has $y_{2}>Y_{1}$ for all $\lambda$ excepting for the situation where $y_{2}=Y_{1}$ which occurs for the special value

$$
\lambda=\left(N_{c} / N_{0}-1\right)^{2 / 3} \text {. }
$$

Since $a, b, N_{0}$ are determined by the logistic model and the initial population, and $t_{1}$ (and hence $\lambda$ ) is fixed, once the time horizon is specified it is possible to compute the values on each side of (15). If they agree then the maximum yield $y_{2}$ for the two-stage process is precisely the same as the maximum yield $y_{1}$ for the one-stage process. Accordingly, if one knew this to be the case then there would be no need to harvest at the time stage $t_{1}$ : one could just wait until $t_{n}$ and harvest everything without losing any individual. However, if (15) is not satisfied (14) indicates that $y_{2}>y_{1}$ and it is better to implement the twostage harvesting process.

It is apparent that the analysis of the previous paragraph can be generalized to deal with $n$ stages. Here $y_{n}$ is defined by (13) and $Y_{n}$ is defined to be the maximum yield when there is no harvesting over the first $n-1$ stages until the last stage at $t_{n}$, and is obtained from $y_{1}$ when $y_{1}$ is expressed in terms of the same value of $\lambda$ as is used in $y_{n}$;

$$
Y_{n}=a N_{0} \lambda^{n} /\left[a+b N_{0}\left(\lambda^{n}-1\right)\right] .
$$

Accordingly the difference

where

$$
y_{n}-Y_{n}=a^{3}\left(\lambda^{1 / 2}-1\right) \Phi(X, \lambda) / D(\lambda),
$$

$$
\begin{gathered}
\Phi(X, \lambda)=f(\lambda) X^{2}+g(\lambda) X+n-1, \quad X=b N_{0} / a, \\
f(\lambda)=\lambda\left(\lambda^{1 / 2}+1\right)\left(\lambda^{n-1}-1\right)\left(\lambda^{1 / 2}-1\right)^{-1}+(n-1)(\lambda-1)\left(\lambda^{n}-1\right)>0, \\
g(\lambda)=(n-1)\left(\lambda^{n}+\lambda-2\right)-\lambda\left(\lambda^{1 / 2}+1\right)\left(\lambda^{n-1}-1\right)\left(\lambda^{1 / 2}-1\right)^{-1}, \\
D(\lambda)=b\left(\lambda^{1 / 2}+1\right)\left[a+b N_{0}(\lambda-1)\right]\left[a+b N_{0}\left(\lambda^{n}-1\right)\right] .
\end{gathered}
$$


Unfortunately it does not appear to be possible to express (16) in a form like that given by the right-hand side of (14). Let $X_{1}, X_{2}$ denote the roots (if any exist) of $\Phi(X, \lambda)=0$, regarded as a quadratic in $X$. There are two cases.

Case $(i) . \quad \mathrm{X}_{1}$ and $\mathrm{X}_{2}$ are distinct (and $\mathrm{X}_{1}<\mathrm{X}_{2}$ ). This situation can occur when the discriminant $g^{2}-4(n-1) f>0$, which may only be true for $\lambda$ greater than some value. It follows that either (A) $\Phi(X, \lambda)>0$ for $X<X_{1}$ and $X>X_{2}$ with $y_{n}>y_{1}$ or (B) $\Phi(X, \lambda)<0$ for $X_{1}<X<X_{2}$ with $y_{n}<y_{1}$, which means that one would avoid implementation of the $n$-stage harvesting strategy or (C) $\Phi(X, \lambda)=0$ for $X=X_{1}$ and $X=X_{2}$ with $y_{n}=y_{1}$ and there is no advantage in performing a harvesting in the $n$-stage process.

Case (ii). $\quad \mathrm{X}_{1}$ and $\mathrm{X}_{2}$ not distinct. In this event $\Phi(X, \lambda)>0$ and $y_{n}>y_{1}$ unless $X=X_{1}$ in which situation $y_{n}=y_{1}$. This would correspond to the graph in Figure 2.

Given any harvesting situation of the kind under consideration here, it appears that one should actually compute numerical values for the maximum yields. A direct comparison of these numbers must reveal whether one should wait for some later time before harvesting.

Initial Population Greater than the Carrying Capacity. Consider a situation in which the initial population $N_{0}$ is greater than the carrying capacity $N_{c}$. Say at some later time $t_{*}$ it is desired to harvest a certain number from the population. If $k_{*}$ denotes the influence variable then the yield is $k_{*} N_{*}$ at $t_{*}$. This leaves $\left(1-k_{*}\right) N_{*}$ and one would desire this to be equal to some value less than $N_{c}$, but not small enough to invalidate the continuous time model. Consequently it seems reasonable to set

$$
\left(1-k_{*}\right) N_{*}=N_{\text {crit }}
$$

where $N_{\text {crit }}$ is the minimum number in the population necessary to allow the validity of writing $\dot{N}$. Once the population is at $N_{\text {crit }}$ then the species will grow and the harvesting strategies discussed earlier in this paper will be applicable. Of course the sooner one can decrease the level below $N_{c}$ the better. Thus one need not wait until $t_{*}$ to harvest; the level could be reduced to $N_{\text {crit }}$ at the initial time $t_{0}$.

Discrete Time Logistic Growth Models. The following list (which certainly is not complete) of nonlinear difference equations produces monotonic increasing behavior of the logistic type for a single species:

$$
\text { (A) } N(t+h)=(1+a h) N(t) /[1+b h N(t)], \quad N=N_{0} \text { at } t=t_{0} \text {, }
$$


with the analytic solution

$$
\begin{aligned}
N(t) & =\frac{a N_{0}(1+a h)^{\left(t-t_{0}\right) / h}}{a+b N_{0}\left[(1+a h)^{\left(t-t_{0}\right) / h}-1\right]}, \\
\text { (B) } N(t+h) & =(1+a h) c^{1-\alpha} N(t) /\left\{c^{1-\alpha}+\alpha b h[N(t)]^{\alpha}\right\}, \\
\text { (C) } N(t+h) & =\left\{1+a h-\alpha b h c^{\alpha-1}[N(t)]^{\alpha}\right\} N(t), \\
\text { (D) } N(t+h) & =\left[1+a h-b h N(t)+c h^{2} N^{2}(t)\right] N(t), \\
\text { (E) } N(t+h) & =N(t) \exp \left\{r h\left[1-N(t) / N_{c}\right]\right\}
\end{aligned}
$$

in each of the above five equations $a, b, c, \alpha$ and $r$ are positive constants. (This last equation was suggested to the author by Prof. R. May.) The last four equations do not appear to have an analytic solution. For $\alpha=1$, it is straightforward to verify, in the limit as the time step $h \rightarrow 0$, that each equation directly reduces to (1).

Consider the model (17). Upon comparing the mathematical forms in (2) and (18) it is evident that the $n$-stage harvesting policy can be implemented directly by just a slight change of notation. For example, the influence variables (regarded as being continuous) are

$$
k_{1}=1-a / b\left(\mu^{1 / 2}+1\right) N_{1}, \quad k_{2}=k_{3}=\cdots=k_{n-1}=1-\mu^{-1 / 2},
$$

the population levels (before harvesting) at the stages $2,3, \ldots, n$ are

$$
N_{2}=N_{3}=\cdots=N\left(t_{n}\right)=a \mu^{1 / 2} / b\left(\mu^{1 / 2}+1\right),
$$

and the maximum yield is

$$
y_{n}=N_{1}+(n-1) a\left(\mu^{1 / 2}-1\right) / b\left(\mu^{1 / 2}+1\right),
$$

where $\mu=(1+a h)^{\left(t-t_{0}\right) / h}$. Other results, corresponding to (15) etc., can be obtained by replacing $\lambda$ with $\mu$.

Influence Variable Discrete. If the influence variable is assumed to be discrete then the maximization of the yield can be obtained by computing the yield corresponding to each $k$ value, comparing the resulting numbers, and then placing them in order.

If the population dynamics model is sufficiently complicated then one may be coerced into using sophisticated one-, two-, or multi-variable search procedures, such as described in Gottfried and Weisman (1973), or one may care to use dynamic programming (Bellman, 1957).

Alternative Differential Equation Models. It is possible to implement the Elizarov and Svirezhev strategy for other growth equations and in the Appendix are 
stated the results for the Gompertz equation and for one biologically realistic equation which is a generalization of the logistic growth equation.

Optimal Control. Whereas the maximum yield by the Elizarov and Svirezhev strategy is obtained by continuously harvesting the species it is not readily apparent what the actual harvest rate should be. In order to gain further insight into this situation the following problem is posed: Maximize the total yield (take) when a species, growing according to the continuous time logistic equation, is being harvested continuously at a rate $u$ over a finite time interval of length $T$. Mathematically, our objective is to maximize

$$
\int_{0}^{T} \dot{u} \mathrm{~d} t
$$

subject to the differential constraint $\dot{N}=(a-b N) N-\dot{u}, N=N_{0}$ at $t=0$. This mathematical problem falls within the category of optimal control theory. The methods described, for example, in Gottlieb and Weisman (1973, p. 375), are applicable here and one finds that, for $N=a / 2 b$, when one continuously harvests at the constant rate $\dot{u}=a^{2} / 4 b$ then the maximum take is $a^{2} T / 4 b$. It is interesting to note that an identical quantity appears in the expression $N_{0}+$ $a^{2} T / 4 b$, yet the problem just posed is not identical with the Elizarov and Svirezhev one.

There is some intrinsic interest in studying the problem of the continuous harvesting (or control) of a species over a fixed time interval of length $T$ when there is a constant rate of removal of members from the population. Consider

$$
\begin{gathered}
\dot{N}=G(N, K)=-K+a N-b N^{2}, \\
N=N_{0} \quad \text { at } t=0, \quad N_{\text {crit }} \leq N_{0}, \quad K>0,
\end{gathered}
$$

where $K$ denotes a constant rate of removal of members of the population and $G(N, K)$ is introduced for convenience; $N>0$. Define the quantities

$$
\begin{aligned}
\alpha & =\left(a^{2}-4 K b\right)^{1 / 2}, \quad \beta=\left(4 K b-a^{2}\right)^{1 / 2}, \\
N_{1} & =(a-\alpha) / 2 b, \quad N_{2}=(a+\alpha) / 2 b .
\end{aligned}
$$

Useful information on the sign of $\dot{N}$ is obtained by constructing a phase diagram and it is apparent that there are three cases according as $K>$, or $=$, or $<a^{2} / 4 b$.

Case $\mathrm{K}>\mathrm{a}^{2} / 4 \mathrm{~b}$. Here $\dot{N}<0$ for all values of $N$, and (21) integrates to give

$$
N(t)=\frac{a}{2 b}-\frac{\beta}{2 b} \tan \left(\frac{\beta}{2} t-C\right), \quad N_{0}=\frac{a}{2 b}-\frac{\beta}{2 b} \tan C .
$$

These equations reveal that $N$ is reduced from $N_{0}$ either to the level $N(T)$ by the time $T\left(<t_{c}\right)$, where $N(T)>N_{\text {crit }}$, or to the level $N_{\text {crit }}$ at $t_{c}(<T)$, where 
$N(T)<N_{\text {crit }}$; here $t_{c}$ is the time, derived from (22), for $N$ to be reduced to $N_{\text {crit }}$ from its initial size $N_{0}$. (If $T=t_{c}$ then $N(T)=N_{\text {crit }}$.) Although our model cannot be valid for $N<N_{\text {crit }}$ it is nevertheless tempting to assume that it is, for then (22) gives the time to extinction $t_{e}$ of the species:

$$
t_{e}=(2 / \beta)[C+\arctan (\alpha / \beta)] .
$$

Case $\mathrm{K}=\mathrm{a}^{2} / 4 \mathrm{~b} . \quad$ Equation (21) becomes

$$
\dot{N}=-b(N-a / 2 b)^{2} \text {. }
$$

There are two sub-cases according as $\dot{N}<0$ for all $N$ excepting $N=a / 2 b$, and $\dot{N}=0$ for $N=a / 2 b$.

(A) $\dot{N}<0(N \neq a / 2 b)$. There are two further sub-cases:

(i) $N>a / 2 b$ : Equation (23) integrates to give

$$
N(t)=a / 2 b+\left[b t+\left(N_{0}-a / 2 b\right)^{-1}\right]^{-1},
$$

and $N$ decreases monotonically from $N_{0}$ to $a / 2 b$. The bound $a / 2 b$ occurs as $t \rightarrow \infty$ and in the limit $\dot{N}$ approaches zero. Note that there are $N(T)$ individuals left in the population at the time $T$.

(ii) $N_{\text {crit }}<N_{0}<N<a / 2 b$ : Equation (23) integrates to give

$$
N(t)=a / 2 b-\left[\left(a / 2 b-N_{0}\right)^{-1}-b t\right]^{-1},
$$

and the population decreases monotonically from $N_{0}$ either to $N_{\text {crit }}$, if $t_{c}<T$ or to $N(T)$, if $T<t_{c}$.

(B) $\dot{N}=0, N=a / 2 b$. Here $N=N_{0}=$ constant and $N(T)=N_{0}=a / 2 b$. This is an equilibrium situation and gives rise to the maximum harvest $a^{2} T / 4 b$.

For completeness it is necessary to provide a discussion on the stability of this equilibrium population. Accordingly, set

$$
N(t)=a / 2 b+\epsilon(t), \quad \epsilon(t)>0, \quad \epsilon(0)=\epsilon_{0},
$$

where $\epsilon(t)$ is a perturbation of the equilibrium number $a / 2 b$. One interpretation of $\epsilon(t)$ is that it represents members of the same species being introduced. Substitution of (24) into (23) gives

$$
\epsilon(t)=\left(b t+\epsilon_{0}^{-1}\right)^{-1}, \quad \dot{\epsilon}<0
$$

and hence $\epsilon(t) \rightarrow 0$ as $t \rightarrow \infty$, indicating that the equilibrium population $a / 2 b$ is asymptotically stable to positive perturbations. However if

$$
N(t)=a / 2 b-\epsilon(t), \quad \epsilon(t)>0, \quad \epsilon(0)=\epsilon_{0},
$$

where $\epsilon(t)$ can be interpreted as the number of individuals being removed at $t$ because of emigration, death, etc. then this time

$$
\epsilon(t)=\left(\epsilon_{0}^{-1}-b t\right)^{-1}, \quad \dot{\epsilon}>0 .
$$


The perturbation increases with time and when $\epsilon(t)=a / 2 b-N_{\text {crit }}$ then $N(t)=N_{\text {crit }}$, the lower level for validity of our model. Accordingly, one would then cease harvesting until the population recovered.

Case $\mathrm{K}<\mathrm{a}^{2} / 4 \mathrm{~b}$. (A) $N_{\text {crit }}<N_{1}<N<N_{2}$ : For this range of $N, \dot{N}>0$ and

$$
N(t)=\frac{\left(N_{2}-N_{0}\right) N_{1}+\left(N_{0}-N_{1}\right) N_{2} \mathrm{e}^{\alpha t}}{N_{2}-N_{0}+\left(N_{0}-N_{1}\right) \mathrm{e}^{\alpha t}}
$$

as $t \rightarrow \infty, N \rightarrow N_{2}$. The population increases monotonically from $N_{0}$ to $N_{2}$ and is at the level $N(T)$ at time $T$.

(B) $N_{\text {crit }}<N<N_{1}$. In this interval $\dot{N}<0$ and $N$ decreases from $N_{0}$ to $N_{\text {crit }}$.

(C) $N>N_{2}$. The population decreases monotonically from $N_{0}$ to the level $N_{2}$.

Many of the details of this section are not in agreement with the work of Goh (1969). For example, he has no qualms about discussing $\dot{N}$, and drawing conclusions concerning harvesting to extinction, when $N$ is small enough, so that $\dot{N}$ can have no meaning.

Possible Biological Applications. Goh (1969/70) suggests the use of (1) in connection with harvesting a fisheries resource, and Clark (1973) has used (1) in his studies of maximizing the economic rent (instead of the biological yield) derived from the sale of the Antarctic blue whale. These authors do not substantiate their use of (1) with any biological data. Elizarov and Svirezhev (1972) do present a discussion of the culling of microorganisms in a chemostat. An interesting biological application, in the spirit of the present paper, is provided by Miller and Botkin (1974).

Conclusions and Discussion. If one restricts attention to the implementation of the Elizarov and Svirezhev $n$-stage harvesting strategy then the fixed time horizon $T$ and the parameters $a, b, N_{0}$ (from the logistic growth model) fix the levels of population at the times $t_{2}, t_{3}, \ldots, t_{n}$ to be one of three curves; see Figure 1 for the 3-stage process. It is interesting that, mathematically, one can obtain explicit expressions for the influence variables in the case when each is assumed to be continuous.

In order to deal with the matter of knowing beforehand whether a maximum take for an $n$-stage process will actually be greater than the maximum take for the $n$ - 1-stage process it seems that one has actually to compute these two numbers and compare them.

After this paper was written, a referee pointed out related papers of Russian 
work, especially those by Elizarov and Rybalko (1970), Elizarov and Svirezhev (1972a) and Svirezhev and Elizarov (1972).

Although some of the ingredients of optimal control theory are introduced, no mathematical proofs are presented to say precisely in what sense the solutions obtained are optimal. Rather, the author's approach has been to give plausibility to the arguments:

When optimal control theory is applied to a continuous time logistic growth model with a constant rate of harvesting $K$ our findings are as follows: (a) If $K=K_{1}\left(>a^{2} / 4 b\right)$ and $N_{0}>a / 2 b$, then harvest at the rate $K_{1}$ until the population level $a / 2 b$ is reached. Now reduce $K_{1}$ to $K_{*}=a^{2} / 4 b$ and harvest at this rate. If the population drops below $a / 2 b$ harvesting should cease until the species can recover. (b) If $K=K_{*}\left(=a^{2} / 4 b\right)$, then one should harvest continuously at this rate with the proviso that, when the number of individuals drops below $a / 2 b$, there be no harvesting until the population recovers. (c) If $K=K_{2}\left(<a^{2} / 4 b\right)$, then sub-optimal yields are obtained. In practice this may be desirable. For example, one may wish to harvest a number of trees, but leave enough for aesthetic reasons.

\section{APPENDIX}

Other Differential Equation Models. Attempts at generalizing the differential equation (1) tend to give rise to equations which do not appear to possess analytical solutions. One possible generalization is

$$
\dot{N}=\left(a c^{1-\alpha}-\alpha b N^{\alpha}\right) N c^{\alpha-1}, \quad N=N_{0} \quad \text { at } t=t_{0},
$$

where $a, b$ are defined as before, $c$ and $\alpha(>0)$ are constants; $\alpha=1$ gives (1). A literature search by the author revealed that (25) is suggested, in some situations, as a viable alternative to continuous time logistic growth, by Ayala, Gilpin and Ehrenfeld (1973), Gilpin and Ayala (1973). The quantity $\alpha$ allows for asymmetry of the population profiles in the $(N, t)$ plane. As $h \rightarrow 0$ the difference equation models (19) and (20) reduce to (25). Equation (25) has the solution

$$
N(t)=\left[\frac{a c^{1-\alpha} N_{0}^{\alpha} \exp \left[\alpha a\left(t-t_{0}\right)\right]}{a c^{1-\alpha}+\alpha b N_{0}^{\alpha}\left\{\exp \left[\alpha a\left(t-t_{0}\right)\right]-1\right\}}\right]^{1 / \alpha} .
$$

There is no difficulty in carrying through the details of the Elizarov and Svirezhev strategy. The results are:

$$
\begin{gathered}
k_{1}=1-\left[\frac{a c^{1-\alpha}(V-1)}{a b N_{1}^{\alpha}(\lambda-1)}\right]^{1 / \alpha}, \quad k_{2}=k_{3}=\cdots=k_{n-1}=1-\frac{1}{V}, \\
N_{2}=N_{3}=\cdots=N\left(t_{n}\right)=\left[a c^{1-\alpha} \lambda(V-1) /(\lambda-1) V \alpha b\right]^{1 / \alpha}, \\
y_{n}=N_{1}+(n-1)(V-1)\left[a c^{1-\alpha}(V-1) / \alpha b(\lambda-1)\right]^{1 / \alpha},
\end{gathered}
$$

where $\lambda=\exp \alpha a\left(t_{n}-t_{n-1}\right), V=\lambda^{1 /(1+\alpha)}$. Define

$$
\Lambda(\lambda)=c^{-\alpha}(V-1)^{1 / \alpha}\left[a c^{1-\alpha}+\alpha b N_{0}^{\alpha}(\lambda-1)\right]^{1 / \alpha}-[V \alpha b(\lambda-1)]^{1 / \alpha}
$$

then ${ }_{2}>$, or $=$, or $\left\langle N_{1}\right.$ according as $\Lambda(\lambda)>$, or $=$, or $<0$, and the situation is charac- 
terized by a set of three curves similar to those shown in Figure 1 where $\alpha=1$. The maximum yield under continuous harvesting is

$$
N_{0}+\left(a c^{1-\alpha} / \alpha b\right)^{1 / \alpha} \alpha a T /(1+\alpha)^{(1+\alpha) / \alpha} .
$$

Gompertz (1825) introduced the continuous time equation

$$
\dot{N}=r N \ln \left(N_{c} / N\right), \quad N=N_{0} \text { at } t=t_{0},
$$

to model human mortality; $N_{c}$ is the carrying capacity. When $r>0$ this equation can be used to model the growth of tumors (Laird, 1964). The solution is

$$
N(t)=N_{c} \exp \left\{-\exp \left[-r\left(t-t_{0}\right)\right] \ln \left(N_{c} / N_{0}\right)\right\} .
$$

The Elizarov and Svirezhev strategy, for $\nu=\exp \left[-r\left(t_{n}-t_{n-1}\right)\right]$, gives

$$
\begin{gathered}
k_{1}=1-\left(N_{c} / N_{1}\right) \nu^{1 /(1-v)}, \quad k_{2}=k_{3}=\cdots=k_{n-1}=1-v, \\
N_{2}=N_{3}=\cdots=N\left(t_{n}\right)=N_{c} \nu^{v /(1-v)}, \\
N_{2}-N_{1}=\left[\nu^{v /(1-v)}-\left(N_{c} / N_{0}\right)^{-v}\right] N_{c},
\end{gathered}
$$

with growth characteristios similar to those shown in Figure 1. The maximum yield under continuous harvesting is

$$
N_{0}+r T N_{c} e^{-1}
$$

\section{LITERATURE}

Ayala, F. J., M. E. Gilpin and J. G. Ehrenfeld. 1973. "Competition Between Species: Theoretical Models and Experimental Tests." Theo. Pop. Biol., 4, 331-356.

Becker, N. G. 1970. "Control of a Pest Population." Biometrics, 26, 365-375.

Bellman, R. E. 1957. Dynamic Programming. Princeton: Princeton University Press. - and R. Kalaba. 1960. "Some Mathematical Aspects of Optimal Predation in

Ecology and Boviculture." Proc. Natn. Acad. Sci. U.S.A., 46, 718-720.

Cherniavsky, E. A. and H. M. Taylor. 1972. "Control of a General Lethal Growth Process," Math. Biosci., 13, 235-252.

Clark, C. W. 1973. "The Economics of Overexploitation." Science, 181, 630-634.

D'ans, G., P. Kokotović and D. Gottlieb. 1971. "Time-Optimal Control for a Model of Bacterial Growth." J. Optimization Theory Appl., 7, 61-69.

Elizarov, E. Ya. and A. Rybalko. 1970. "Maximal Productivity in Homogeneous Population." Studia Biophysica (Berlin), No. 23, 43-50.

and Yu. M. Svirezhov. 1972. "The Optimal Productivity of Biogeocenoses." Systems Theory Res., 22, 193-204.

- and 1972a. "On Optimal Productivity of Biosystems." Zhurnal Obshchey Biologii., 33, 251-260.

Gilpin, M. E. and F. J. Ayala. 1973. "Global Models of Growth and Competition." Proc. Natn. Acad. Sci. U.S.A., 70, 3590-3593.

Goh, B. S. 1969/1970. "Optimal Control of a Fish Resource." Malayan Scientist, 5, $65-70$.

Gompertz, B. 1825. "On the Nature of the Function Expressive of the Law of Human Mortality and on a New Model of Determining Life Contingencies." Phil. Trans. Roy. Soc. (Lond.), 115, 513-585.

Gottfried, B. S. and J. Weisman. 1973. Introduction to Optimization Theory. Englewood Cliffs: Prentice-Hall.

Jaquette, D. L. 1972. "Mathematical Models for the Control of Growing Biological Populations." Operations Res., 20, 1142-1151. 
Laird, A. K. 1964. "Dynamics of Tumor Growth." Br. J. Cancer, 18, 490-502.

Miller, R. S. and D. B. Botkin. 1974. "Endangered Species: Models and Predictions." Am. Sci., 62, 172-181.

Pearl, R. 1924. Studies in Human Biology. New York: Williams \& Wilkins.

Shoemaker, C. A. 1973. "Optimization of Agricultural Pest Management III. Results and Extensions of a Model." Math. Biosci., 18, 1-22.

Svirezhev, Yu. M. and E. Ya. Elizarov. 1972. "Mathematical Modeling of Biological Systems." In Problems of Space Biology, Vol. 20. Moscow: Nauka.

Verhulst, P. F. 1845. "Recherches Mathématiques sur la loi d'Acroissement de la Population." Mem. Acad. Roy. Bruxelles, 18, 1.

-1. 1847. "Recherches Mathématiques sur la loi d'Acroissement de la Population." Mem. Acad. Roy. Bruxelles, $20,1$.

RECEIVED 11-20-74

REVISED $\quad 5-8-75$ 